\title{
Orientación de nidos de hornero (Furnarius rufus): Efectos de la vegetación, el viento y la radiación solar en el noroeste de la Argentina
}

\author{
AlejandRo A. SchaAF \\ Instituto de Ecorregiones Andinas (INECOA), Universidad Nacional de Jujuy - Consejo Nacional de Investigaciones \\ Científicas y Técnicas (CONICET), San Salvador de Jujuy, Jujuy, Argentina.
}

\begin{abstract}
Resumen. La radiación solar, los vientos y la cobertura vegetal sobre los nidos pueden influir en las decisiones de las aves acerca de cómo construirlos; por ejemplo, en la orientación de la entrada. En este estudio se examinó la orientación de la entrada en nidos de hornero (Furnarius rufus) en un sitio desértico subtropical del norte de la Argentina. Se evaluó si la radiación solar y los vientos influyen en la orientación media de los nidos con abundante cobertura vegetal superior y en los que tienen escasa o nula cobertura vegetal superior. Los nidos con abundante cobertura vegetal superior mostraron una orientación aleatoria de la boca de entrada, lo que sugiere que la radiación solar y los vientos no influyen al momento de la construcción del nido. Los nidos con poca o nula cobertura vegetal superior presentaron una orientación de entrada no aleatoria hacia el sureste, con lo cual reducen la radiación solar directa. Además, estos nidos con escasa cobertura vegetal se orientan hacia los vientos húmedos predominantes del sur, lo que facilitaría la refrigeración dentro del nido. Este trabajo resalta la importancia de incluir la cobertura vegetal de los nidos como variable que puede influir en la orientación de los nidos.
\end{abstract}

[Palabras clave: desierto, Furnariidae, neotrópico, orientación del nido, Passeriformes]

\begin{abstract}
AвSTRACt. Nest orientation of rufous hornero (Furnarius rufus): Vegetation, wind and solar radiation effects in Northwestern Argentina. Solar radiation, winds and cover vegetation can affect nest construction decisions in birds; for example, the orientation of their entrance. In this study, the nest entrance orientation of rufous hornero (Furnarius rufus) in a subtropical desert site from Northern Argentina was examined. It was evaluated whether solar radiation and winds influence the mean orientation of nests with abundant upper vegetation cover and of those with little or no upper vegetation cover. Nests with abundant upper vegetation cover showed a randomly defined nest entrance orientation, suggesting that solar radiation and winds are not relevant in nest construction. Nests with little or no upper vegetation cover showed a non-random entrance, southeastern oriented, which would avoid direct solar radiation. Thus, nest with little or no upper vegetation cover were oriented towards prevailing southern humid winds as to increase cooling inside the nest. This work emphasizes the importance of including nest vegetation cover as a variable that may affect nest orientation.
\end{abstract}

[Keywords: desert, Furnariidae, neotropic, nest birds orientation, Passerines]

\section{INTRODUCCIÓN}

Las condiciones meteorológicas tales como el viento, la lluvia y la radiación solar pueden influir en el comportamiento de construcción de los nidos de las aves (Facemire et al. 1990; Hartman and Oring 2003; Mezquida 2004; Burton 2006; Schaaf et al. 2018a). En particular, la radiación solar puede beneficiar a las aves de climas templados o fríos, ya que les ayuda a mantener temperaturas cálidas dentro de los nidos ante la ocurrencia de bajas temperaturas diurnas y nocturnas; mientras tanto, las aves de climas tropicales o subtropicales pueden verse perjudicadas por el sobrecalentamiento de los nidos durante la etapa reproductiva (Burton 2007; Landler et al. 2014; Mainwaring et al. 2016). Trabajos realizados en el hemisferio norte encontraron que en sitios cercanos al ecuador, las aves pueden evitar el sobrecalentamiento de los nidos orientando sus nidos hacia el norte o al este-oeste, donde la exposición solar es menor (Wiebe 2001; Burton 2007; Landler et al. 2014). Otra manera de evitar esta sobreexposición solar es colocar los nidos en sitios protegidos por abundante cobertura vegetal superior (Souza and Santos 2007; Schaaf et al. 2018b).

La orientación del nido puede estar correlacionada con los vientos predominantes del sitio para facilitar la refrigeración dentro del nido (Ricklefs and Hainsworth 1969; Facemire et al. 1990; Viñuela and Sunyer 1992; Norment 
1993; Mezquida 2004). En ambientes desérticos, algunas especies de aves que construyen nidos cerrados pueden evitar la incidencia directa del viento cuando las temperaturas son más bajas $\mathrm{y}$, a medida que avanza la temporada reproductiva y las temperaturas ambientales aumentan, orientan la boca hacia los vientos dominantes (Mezquida 2004). De esta manera logran microclimas óptimos dentro del nido para garantizar un mayor éxito reproductivo (Ricklefs and Hainsworth 1969; Facemire et al. 1990; Mezquida 2004).

El hornero (Furnarius rufus) es una especie que construye nidos cerrados y orienta la boca de entrada de sus nidos según el grado de protección por cobertura vegetal superior (Souza and Santos 2007; Schaaf et al. 2018b). Schaaf et al. (2018b) demostraron que la orientación de la entrada de los nidos con abundante cobertura vegetal sobre el nido fue al azar en la mayoría de las localidades estudiadas, ya quela cobertura vegetal superior les otorga beneficios de protección contra la radiación solar. Mientras tanto, los nidos con escasa o nula vegetal superior mostraron orientaciones de entradas definidas en cinco de los diez lugares estudiados. Específicamente para San Salvador de Jujuy, a 1260 m s. n. m., estos nidos desprotegidos de la radiación solar presentaron una orientación media hacia el oeste, lo que podría permitir la acumulación de calor durante las horas de la tarde antes de que ocurran las bajas temperaturas nocturnas. De manera similar, en un gradiente altitudinal en los bosques de Yungas, los nidos con escasa o nula cobertura vegetal se orientaban hacia el noroeste a mayores altitudes (Schaaf, 2020). De esta manera, esta especie de ave podría estar disminuyendo la humedad y el enfriamiento de los nidos en sitios lluviosos con bajas temperaturas nocturnas al orientarlo hacia donde la exposición solar es mayor (Burton 2007; Mainwaring et al. 2016).

A pesar de estos avances en sitios tropicales y subtropicales húmedos, no se analizaron otras variables locales como los vientos del lugar (Schaaf et al. 2018b), y se desconoce qué sucede en regiones áridas subtropicales. Por esta razón, este trabajo se enfoca en analizar la orientación de la boca de entrada de los nidos de esta especie de ave en un ambiente árido altoandino de la provincia de Jujuy, Argentina, con escasas lluvias y alta exposición solar diurna. Teniendo en cuenta la hipótesis de que las temperaturas ambientales y la radiación solar pueden influenciar la orientación de los nidos (Wiebe 2001; Burton 2007; Landler et al. 2014), esperamos que la entrada de los nidos con buena protección vegetal superior se oriente al azar (Schaaf et al. 2018b). A su vez, esperamos que los nidos sin cobertura vegetal superior presenten un patrón definido de orientación, evitando la radiación solar directa proveniente del norte. Debido a que se trata de un sitio subtropical seco y con escasas lluvias, esperamos que los nidos desprotegidos orienten su boca de entrada hacia los vientos húmedos predominantes, con lo que podrían aumentar la humedad dentro del nido (Facemire et al. 1990).

\section{MATERIALES Y MÉTODOS}

El trabajo se llevó a cabo en ambientes urbanos y periurbanos de la localidad de Tilcara, provincia de Jujuy, Argentina $\left(23^{\circ}\right.$ $34.612^{\prime} \mathrm{S}-65^{\circ} 23.602^{\prime} \mathrm{O} ; 2450$ a $2500 \mathrm{~m} \mathrm{~s}$. n. m.) (Figura 1). El clima es característico de los desiertos subtropicales de altura, con una intensa sequedad acentuada por fuerte irradiación solar, con lluvias escasas y estacionales menores a los $200 \mathrm{~mm} / \mathrm{año}$, concentradas entre diciembre y marzo). Las temperaturas medias diurnas durante la primavera y verano son muy altas durante el día y frías durante la noche, pudiendo superar los $25-30{ }^{\circ} \mathrm{C}$ durante la tarde, y descender a menos de $10^{\circ} \mathrm{C}$ durante la noche, dando como resultado una gran amplitud térmica diaria. Los vientos preponderantes durante esta época son húmedos y provienen mayormente del este y sureste (Buitrago 2000; Reboratti 2003). La vegetación está representada principalmente por especies de árboles nativos como Molle (Schinus areira), Algarrobo (Prosopis alba), y especies exóticas como Ciprés (Cupressus sp.) y Álamo (Populus alba) (Reboratti 2003; Martiarena et al. 2011).

El hornero pertenece a la familia Furnariidae, es una especie monógama y residente permanente en los sitios donde se encuentra. Presenta una amplia distribución en América del Sur y es muy común en ambientes urbanos y rurales, donde construye nidos cerrados de barro en árboles o postes de luz, generalmente desde los meses de agosto-septiembre a diciembre-febrero, en la Argentina. Pone entre 2 y 5 huevos cada dos días, que incuban durante unos 17 días; la eclosión es asincrónica, y los pichones permanecen en los nidos durante 24 a 26 días (Fraga 1980; Zyskowski and Prum 1999; Peña 2005; Shibuya et al. 2015). 

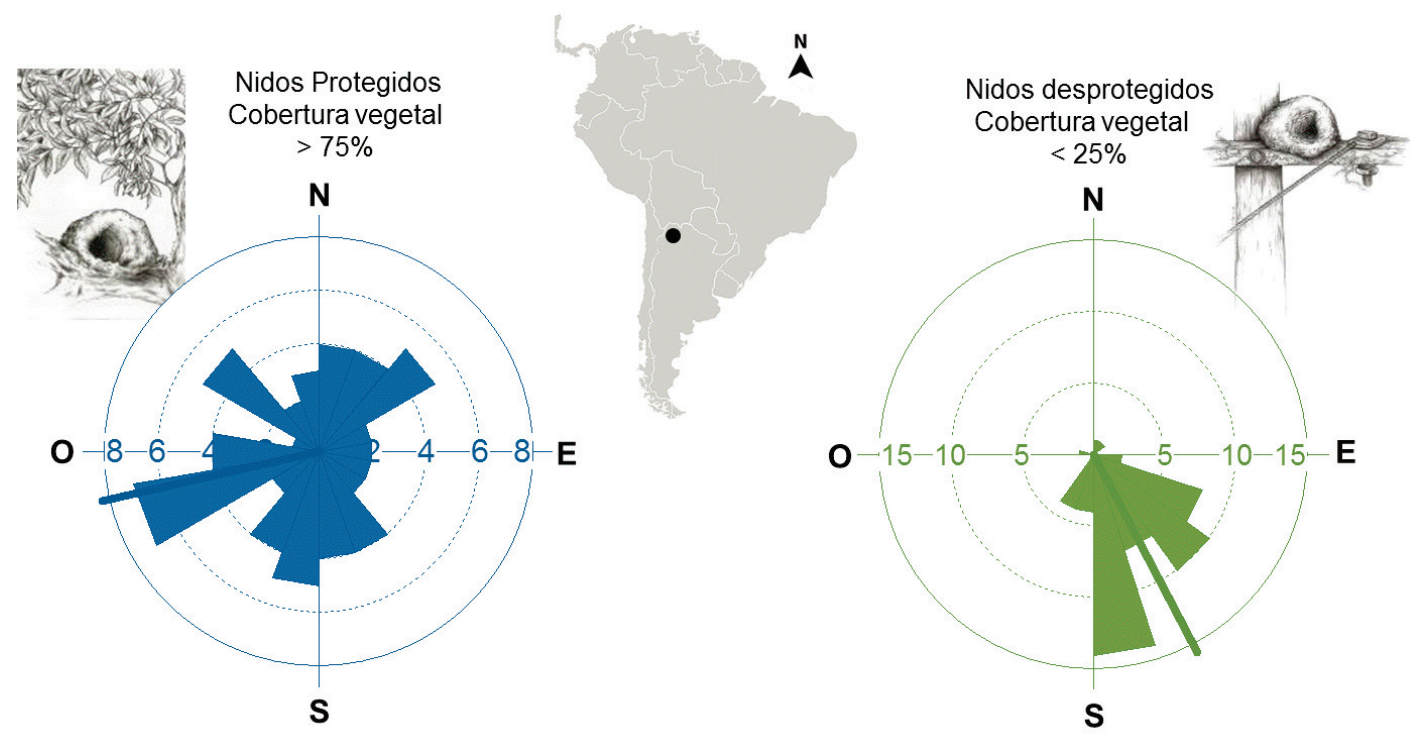

Figura 1. Distribución circular de la boca de entrada de los nidos de hornero (Furnarius rufus) en un área desértica subtropical de altura del norte de la Argentina. Se muestran la orientación media (línea continua) y la cantidad total de observaciones (números en las líneas) para nidos protegidos ( $\mathrm{n}=62$ ) y nidos desprotegidos ( $\mathrm{n}=53$ ).

Figura 1. Circular distribution of rufous hornero (Furnarius rufus) nest entrance in a subtropical desert from Northwestern Argentina. The mean orientation (continuous line) and the total amount of observations (numbers in line) for covered nests $(n=62)$ and uncovered nests $(n=53)$ are shown.

Durante el mes de marzo de 2019 se realizó una búsqueda intensiva de nidos de hornero en árboles y en postes de luz, para lo cual se recorrieron caminos urbanos y periurbanos. Para cada nido encontrado se registró la orientación de entrada del nido usando una brújula, y la cobertura vegetal. La cobertura vegetal se estimó visualmente y fue considerada una variable categórica separada en dos grupos: nidos bien protegidos de la radiación solar directa (cobertura vegetal superior sobre el nido $>75 \%$, ubicados en el interior de los árboles) y nidos con escasa o nula protección vegetal superior (cobertura vegetal superior $<25 \%$, ubicados en árboles muertos en pie y postes de luz) (detalles de la metodología en Schaaf et al. 2018b). Los nidos con una cobertura vegetal entre 25 y 75\% fueron descartados siguiendo la metodología propuesta en Schaaf et al. (2018b), lo que permitió establecer dos grupos extremos bien definidos de cobertura vegetal. Se tomaron datos de nidos inactivos y de nidos construidos durante la temporada de reproducción de septiembre 2018 a febrero 2019, para lo cual se tuvo en cuenta nuestro conocimiento previo en búsqueda y monitoreo de nidos (Schaaf et al. 2018b). Específicamente, se consideró si fueron construidos durante la temporada reproductiva mencionada (e.g., nidos sin rastros de rotura), y para los nidos en árboles muertos en pie se confirmó que estos estén muertos antes de la construcción de los nidos (árboles muertos en pie sin partes vivas y con corteza de tronco en estado de descomposición avanzada). Los datos de orientación se corrigieron según la declinación magnética del norte verdadero (Evans 2017).

Se estimó la orientación media $( \pm$ D.E.) de la boca de entrada de los nidos para las diferentes coberturas de vegetación utilizando estadísticas circulares. La prueba de Rayleigh (z) se usó para determinar si las bocas de entrada de los nidos se distribuyeron al azar $(P>0.05)$ o presentaron un patrón de orientación $(P<0.05)$ (Zar 1999). Se utilizó una prueba de Watson-Williams (F) para estimar las diferencias en los valores de orientación media según el tipo de cobertura vegetal. Todos los análisis y gráficos fueron realizados con el Software Oriana versión 2.0 (Kovach 2004).

\section{Resultados y Discusión}

Se encontró un total de 115 nidos de hornero con cobertura vegetal $<25 \%$ y $>75 \%$. De este total de nidos, 62 tenían una cobertura vegetal superior $>75 \%$ y 53 con cobertura vegetal superior $<25 \%$. Los nidos con abundante cobertura vegetal superior se encontraban en 
árboles de Schinus areira (80\%), Populus alba (15\%) y Pinus sp. (5\%). La orientación media de la boca de entrada de estos nidos fue de $257^{\circ} \pm 127.16$ (al oeste) y se distribuían al azar $(\mathrm{z}=0.45$ y $P=0.64)$. Los nidos con escasa o nula cobertura vegetal superior se encontraron mayormente en árboles muertos en pie (75\%) y postes de luz (25\%). La orientación media de la boca de entrada de estos nidos fue de $152^{\circ} \pm 41.40$ (al sureste) y se distribuían de manera definida $(\mathrm{z}=31.45$ y $P<0.001$ ) (Figura $1)$. Se encontraron diferencias significativas entre los valores medios de las orientaciones al comparar los tipos de cobertura vegetal superior de los nidos (prueba de WatsonWilliams, $\mathrm{F}=14.79$, P0.001).

Los resultados muestran que la boca de entrada de nidos con abundante cobertura vegetal se distribuye al azar, mientras que los nidos con escasa o nula cobertura vegetal se orientan de manera definida hacia el sureste, apoyando la hipótesis de las temperaturas ambientales y los vientos predominantes planteada en este trabajo. Los resultados indican que la cobertura vegetal superior provee a los nidos de protección contra la radiación solar y los vientos, por lo que los nidos con estas condiciones de vegetación orientan su entrada al azar. Mientras que los nidos con escasa vegetación superior evitan la radiación solar directa en la entrada y aprovechan los vientos húmedos predominantes para favorecer su microclima (aumento de humedad) dentro del nido en ambientes desérticos, lo cual favorecería el desarrollo embrionario (Facemire et al. 1990; Deeming 2016; Martin et al. 2017). Además, este trabajo destaca la importancia de medir e incluir la cobertura vegetal superior como variable que puede influir en la orientación de los nidos, la cual está escasamente estudiada (Hoekman et al. 2002; Souza and Santos 2007; Schaaf et al. 2018).

Además de maximizar la termorregulación dentro delos nidos, las aves deben minimizar el riesgo de depredación (Cox et al. 2013; Fogarty et al. 2017). Por esta razón, deben seleccionar sitios con bajo riesgo de depredación y optimizar las temperaturas para favorecer el éxito reproductivo. Así, la selección de sitios con buena cobertura vegetal puede implicar una serie de decisiones que podrían influir en las condiciones microclimáticas de los nidos al tiempo que minimizan el riesgo de depredación visual (Fogarty et al. 2017; Schaaf et al. 2018a). También cabe señalar que esta especie construye sus nidos con paredes gruesas, principalmente con barro, lo que puede otorgar beneficios térmicos durante la incubación y cría de pichones (Shibuya et al. 2015), en particular durante las horas de la noche, cuando las temperaturas ambientales descienden (Reboratti 2003; Mainwaring et al. 2016). Por lo tanto, recomendamos que trabajos futuros analicen la orientación de los nidos en conjunto con otras variables como las temperaturas internas de los nidos, los períodos de incubación, la construcción de los nidos (tipos de material y espesor de paredes) y el éxito reproductivo entre nidos con diferentes tipos de cobertura vegetal superior.

Agradecimientos. Agradezco a los revisores por su trabajo y comentarios que ayudaron a mejorar este manuscrito. A Marisel Morales por realizar los dibujos de la figura. AAS es becario postdoctoral del CONICET (Consejo Nacional de Investigaciones Científicas y Técnicas).

\section{REFERENCIAS}

Buitrago, L. G., and M. T. Larran. 2000. El clima de la Provincia de Jujuy. Cátedra de Climatología y Fenología Agrícolas, Facultad de Ciencias Agrarias, Universidad Nacional de Jujuy.

Burton, N. H. 2006. Nest orientation and hatching success in the Tree Pipit Anthus trivialis. Journal of Avian Biology 37:312-317. https://doi.org/10.1111/j.2006.0908-8857.03822.x.

Burton, N. H. 2007. Intraspecific latitudinal variation in nest orientation among ground-nesting passerines: a study using published data. The Condor 109:441-446. https://doi.org/10.1093/condor/109.2.441.

Cox, W. A., F. R. Thompson III, and J. L. Reidy. 2013. The effects of temperature on nest predation by mammals, birds, and snakes. The Auk 130(4):784-790. https:/ / doi.org/10.1525/auk.2013.13033.

Deeming, D. C. 2016. How does the bird-nest incubation unit work? Avian Biology Research 9(2):103-113. https: //doi.org/10.3184/175815516X14567543242701.

Evans, S. W. 2017. The effect of nest site orientation on the breeding success of Blue Swallows Hirundo atrocaerulea in South Africa. African Journal of Ecology 56:91-100. https://doi.org/10.1111/aje.12421.

Facemire, C. F., M. E. Facemire, and M. C. Facemire. 1990. Wind as a factor in the orientation of entrances of Cactus Wren nests. The Condor 92:1073-1075. https://doi.org/10.2307/1368745.

Fogarty, D. T., R. D. Elmore, S. D. Fuhlendorf, and S. R. Loss. 2017. Influence of olfactory and visual cover on nest 
site selection and nest success for grassland-nesting birds. Ecology and Evolution 7:6247-6258. https://doi.org/ 10.1002/ece3.3195.

Fraga, R. M. 1980. The breeding of rufous hornero (Furnarius rufus). The Condor 82(1):58-68. https:/ / doi.org/10.2307/ 1366785.

Greeney, H. F. 2009. Nest orientation of the Spotted Barbtail, Premnoplex brunnescens, is strongly correlated with stream flow. Journal of Ethology 27(2):203-208. https://doi.org/10.1007/s10164-008-0104-1.

Hartman, C. A., and L. W., and Oring. 2003. Orientation and microclimate of Horned Lark nests: the importance of shade. The Condor 105:158-163. https://doi.org/10.1093/condor/105.1.158.

Hoekman, S. T., I. J. Ball, and T. F. Fondell. 2002. Grassland birds orient nests relative to nearby vegetation. The Wilson Journal of Ornithology 114(4):450-457. https:/ / doi.org/10.1676/0043-5643(2002)114[0450:GBONRT]2.0.CO;2.

Kovach, W. 2004. Oriana v. 2.02 a. Kovach Computing Services, Anglesey, Wales.

Landler, L., M. A. Jusino, J. Skelton, and J. R. Walters. 2014. Global trends in woodpecker cavity entrance orientation: latitudinal and continental effects suggest regional climate influence. Acta Ornithologica 49:257-266. https: / /doi.org/ 10.3161/173484714X687145.

Mainwaring M. C., I. R. Hartley, M. M. Lambrechts, and D. C. Deeming. 2014. The design and function of birds' nests. Ecology and Evolution 4:3909-3928.

Mainwaring, M. C., I. Barber, D. C. Deeming, D. A. Pike, E. A. Roznik, and I. R. Hartley. 2016. Climate change and nesting behaviour in vertebrates: a review of the ecological threats and potential for adaptive responses. Biological Reviews 92(4):1991-2002. https:/ / doi.org/10.1002/ece3.1054.

Martin, T. E., A. J. Boyce, K. FierroロCalderón, A. E. Mitchell, C. E. Armstad, J. C. Mouton, and E. E. Bin Soudi. 2017. Enclosed nests may provide greater thermal than nest predation benefits compared with open nests across latitudes. Functional Ecology 31(6):1231-1240. https:// doi.org/10.1111/1365-2435.12819.

Martiarena, M., S. D. Matteucci, and R. del Sueldo. 2011. Plan de conservación del sistema de espacios verdes urbanos asociados a la red de acequias de riego de la localidad de Tilcara, Jujuy, Argentina. Revista de la Asociación Argentina de Ecología de Paisajes 1:177-190.

Mezquida, E. T. 2004. Patrones de orientación de los nidos de Passeriformes en una zona árida del centro-oeste de Argentina. Ornitologia Neotropical 15:145-153.

Peña, M. R. D. L. 2005. Reproducción de las aves argentinas (con descripción de pichones). LOLA, Buenos Aires.

Reboratti, C. 2003. La quebrada. Geografía, historia y ecología de la Quebrada de Humahuaca. Buenos Aires: La Colmena. Pp. 245.

Schaaf, A. A. 2020. Trends in closed nest entrance orientation of the Rufous Hornero Furnarius rufus along an altitudinal gradient in South America. Bird Study, 1-4.

Schaaf, A. A., T. N. Rojas, A. Díaz, and S. I. Peluc. 2018a. Patterns of nest orientation in the golden billed saltator (Saltator aurantiirostris) in central Argentina. Ornitologia Neotropical 29:199-203.

Schaaf, A. A., C. G. García, P. B. Puechagut, L. E. Silvetti, E. Tallei, F. Ortis, and A. I. Quaglia. 2018b. Effect of geographical latitude and sun exposure on Rufous Hornero (Furnarius rufus) nest orientation. Journal of Ornithology 159(4):967974. https://doi.org/10.1007/s10336-018-1569-5.

Shibuya, F. L., T. V. Braga, and J. J. Roper. 2015. The Rufous Hornero (Furnarius rufus) nest as an incubation chamber. Journal of Thermal Biology 47:7-12. https://doi.org/10.1016/j.jtherbio.2014.10.010.

Souza, F. L, and C. A. Santos. 2007. Climate and nest opening orientation in Furnarius rufus (Furnariidae). Iheringia 97:293-295. https://doi.org/10.1590/S0073-47212007000300013.

Viñuela, J., and C. Sunyer. 1992. Nest orientation and hatching success of Black Kites Milvus migrans in Spain. Ibis 134: 340-345. https://doi.org/10.1111/j.1474-919X.1992.tb08013.x.

Wiebe, K. L. 2001. Microclimate of tree cavity nests: is it important for reproductive success in Northern Flickers? The Auk 118:412-421. https:/ / doi.org/10.1093/auk/118.2.412.

Zar, J. H. 1999. Biostatistical analysis. Prentice Hall, Upper Saddle River.

Zyskowski, K, and R. O. Prum. 1999. Phylogenetic analysis of the nest architecture of Neotropical ovenbirds (Furnariidae). The Auk 116:891-911. https://doi.org/10.2307/4089670. 